\title{
CODES FROM ZERO-DIVISORS AND UNITS IN GROUP RINGS
}

\author{
PAUL HURLEY* AND TED HURLEY ${ }^{\dagger}$
}

\begin{abstract}
We describe and present a new construction method for codes using encodings from group rings. They consist primarily of two types: zero-divisor and unit-derived codes. Previous codes from group rings focused on ideals; for example cyclic codes are ideals in the group ring over a cyclic group. The fresh focus is on the encodings themselves, which only under very limited conditions result in ideals.

We use the result that a group ring is isomorphic to a certain well-defined ring of matrices, and thus every group ring element has an associated matrix. This allows matrix algebra to be used as needed in the study and production of codes, enabling the creation of standard generator and check matrices.

Group rings are a fruitful source of units and zero-divisors from which new codes result. Many code properties, such as being LDPC or self-dual, may be expressed as properties within the group ring thus enabling the construction of codes with these properties. The methods are general enabling the construction of codes with many types of group rings. There is no restriction on the ring and thus codes over the integers, over matrix rings or even over group rings themselves are possible and fruitful.
\end{abstract}

1. Introduction. We present techniques for construction of codes from encodings in group rings, resulting in two new types of codes that we call zero-divisor codes and unit-derived codes. Many existing codes use zero-divisors of special group rings. All cyclic codes, such as BCH, Golay, Hamming and Reed-Solomon are special types of zero-divisor codes of the group ring of the cyclic group.

The use of group rings for the construction of codes has, to date, been concerned with the ideals contained within. As cyclic codes are ideals in the group ring over the cyclic group, this has lead to considering the natural generalisation of cyclic codes as being ideals (e.g. [8]). Indeed, a group ring code has been defined (e.g. [6]) as an ideal in a group ring. A group ring is often called a group algebra when the ring is a field.

Codes from group ring encodings, presented here, are submodules in the group ring and only in certain restrictive cases are they ideals in a group ring. Indeed the unit-derived codes are never ideals. The methods for obtaining generator and check matrices apply in all cases.

We use an isomorphism between a group ring and a certain ring of matrices to enable the straightforward production of check and generator matrices for the codes directly from zero-divisors and units. The interplay between the group ring construction and the corresponding matrix construction is extremely fruitful, allowing immediate calculation (algebraically) of generator and check matrices for these codes.

Looking at group ring encodings expands the space of possible codes, and, we believe, offers a simple and intuitive approach

Properties of codes from group ring encodings, such as self-duality or having a sparse parity-check matrix (namely LDPC) often have an easy algebraic description as properties in zero-divisor or unit-derived codes within group rings and this description can be exploited for the construction and analysis of these examples. We present a number of examples of these LDPC and self-dual zero-divisor and unit-derived codes.

It is also possible to obtain convolutional codes from unit-derived and zero-divisor codes from group rings and this is the subject of a companion work. Also of note is that the underlying algebraic structure of group rings often allows the calculation of distance directly.

The explicit examples presented are necessarily of a short length. The methods, however, are completely general and can be used to construct codes with longer lengths. The algebra can then be used to derive properties of these codes or to construct long length codes with some desired property.

1.1. Overview and layout. We begin, in Section 2, with a description of the basic algebra, defining a group ring $R G$ and zero-divisors and units. We use the result that $R G$ is isomorphic to a certain ring of $n \times n$ matrices over the ring $R$ where $n$ is the order of the group $G$ - see [7]. These matrices are called $R G$-matrices over $R$. This connection is used later to derive generator and check matrices. Examples of $R G$-matrices for different groups $G$ are given - these include circulant matrices, block-type circulant matrices, Toeplitz-type matrices, and Walsh-Toeplitz matrices.

Section 3 defines a code from a group ring encoding. In Section 4, the procedure for obtaining zero-divisor codes is presented, showing how generator and check matrices may be obtained.

*IBM Research, Zurich Research Laboratory, Switzerland. pah@zurich.ibm.com

† National University of Ireland, Galway, Ireland. ted.hurley@nuigalway.ie 
Then the concept of unit-derived codes, which are particularly elegant and simple, is described in Section 5. Obtaining generator and check matrices from units is straightforward, there is much flexibility from which to generate the code, and the invertibility of units offers particular promise for exploitation.

The algebraic group ring descriptions enable new codes and series of codes with particular properties or of a particular type to be constructed. The group ring description may often be used to analyse, or deduce properties of, the codes.

There is no restriction on the ring $R$ in the group ring $R G$ and what is required is either a zero-divisor or a unit within the group ring. Thus, the construction of codes over systems other than fields is possible. In particular, integral codes together with their generator and check matrices may be constructed using units in group rings over the integers.

Example codes are shown in Section 6, starting with some illustrative self-dual codes. Examples of codes when working in group rings over dihedral groups are then given in Section 6.2 (for example an explicit construction of a $(62,30,12)$ code, which is one of best known distance). Using encodings directly and the $R G$-matrix structure, as shown in this work, is new. Previous examinations of codes using dihedral groups worked exclusively using ideals.

In Section 6.3 we describe examples of codes designed to have a sparse check matrix - LDPC (Low Density Parity Check) codes. Most techniques to date obtain LDPC codes by randomisation, but there are recent developments using algebraic techniques (which have advantages such as immediate generator matrices and structure to exploit). We show that some of these implicitly operate in a group ring. Then we propose a regular LDPC code from a group ring of a direct product of groups - from either a zero-divisor or unit-derived code.

Relationships to well-known codes is explored in Section 7. For example, we show that when we specialise to the zero-divisor codes of group rings on cyclic groups which are ideals we get the cyclic or polynomial codes; one is not restricted to ideals and so even in the group ring of the cyclic group, the zero-divisors codes can define codes other than those which are termed cyclic. The (restrictive) conditions under which one of these codes (for general group rings) is an ideal in the group ring are also derived in this section.

2. Group rings. Only basic, necessary definitions and results on group rings are provided. For further information on group rings and related algebra, please see [12].

Let $G$ be a group and $R$ a ring ${ }^{1}$. The group ring $R G$ is a ring consisting of the set of all summations $u=\sum_{g \in G} \alpha_{g} g$, where $\alpha_{g} \in R$. Consider also $v=\sum_{g \in G} \beta_{g} g$. Addition is defined term-by-term,

$$
u+v=\sum_{g \in G}\left(\alpha_{g}+\beta_{g}\right) g,
$$

while multiplication is a convolution-like operation,

$$
u v=\sum_{g, h \in G} \alpha_{g} \beta_{h} g h .
$$

This can be written, since $G$ is a group, as:

$$
u v=\sum_{g \in G}\left(\sum_{h \in G} \alpha_{h} \beta_{h^{-1} g}\right) g .
$$

For example, if $G$ is a finite cyclic group, multiplication is the circulant convolution.

$G$ acts as a basis for the module $R G$ over the ring $R$. In effect, every element of $R G$ is a vector composed of elements of the ring $R$, with the $i$ th component associated with the group element $g_{i}$; addition is of components and multiplication is obtained from the group multiplication together with the distributive law.

The following definition applies in general to any ring but we shall be particularly interested in cases where the ring is a group ring.

\footnotetext{
${ }^{1}$ The ring can be arbitrary; in all cases dealt with here, the ring $R$ has an identity.
} 
Definition 2.1. For any ring $R$ a non-zero element $z \in R$ is a zero-divisor ${ }^{2}$ if and only if there exists a non-zero $r \in R$ such that $z r=0$. For any ring $R$ with identity an element $u \in R$ is a unit if and only if there exists an element $v \in R$ such that $u v=1=v u .{ }^{3}$ When this $v$ exists it is often written as $u^{-1}$.

Sometimes $R$ is a field in the group $\operatorname{ring} R G$, but is not restricted to such, and then $R G$ is called a group algebra. However, other rings such as when $R=\mathbb{Z}$, the integers, cases where $R$ has zero-divisors or where $R$ itself is a group ring, have also proved useful.

2.1. Group rings and matrices. Let $\left\{g_{1}, g_{2}, \ldots, g_{n}\right\}$ be a fixed listing of the elements of $G$. The $R G$-matrix of $w=\sum_{i=1}^{n} \alpha_{g_{i}} g_{i} \in R G$, is in $R_{n \times n}$, the ring of $n \times n$ matrices over $R$, and defined as,

$$
M(R G, w)=\left(\begin{array}{cccc}
\alpha_{g_{1}^{-1} g_{1}} & \alpha_{g_{1}^{-1} g_{2}} & \ldots & \alpha_{g_{1}^{-1} g_{n}} \\
\alpha_{g_{2}^{-1} g_{1}} & \alpha_{g_{2}^{-1} g_{2}} & \ldots & \alpha_{g_{2}^{-1} g_{n}} \\
\vdots & \vdots & \vdots & \vdots \\
\alpha_{g_{n}^{-1} g_{1}} & \alpha_{g_{n}^{-1} g_{2}} & \ldots & \alpha_{g_{n}^{-1} g_{n}}
\end{array}\right)
$$

The first column is, in essence, labelled by $g_{1}$, the second by $g_{2}$ etc.; the first row is in essence labelled by $g_{1}^{-1}$, the second by $g_{2}^{-1}$ etc. Each row and each column is a permutation, determined by the group multiplication, of the initial row.

The first important result, proven in [7], is that a group ring $R G$ is isomorphic to a ring of $R G$-matrices over $R$, a subring of $R_{n \times n}$, the ring of $n \times n$ matrices over $R$.

THEOREM 2.2. Given a listing of the elements of a group $G$ of order $n$ there is a bijective ring homomorphism $\sigma: w \mapsto M(R G, w)$ between $R G$ and the $n \times n R G$-matrices over R..

This result means that the group ring and the ring of matrices are interchangeable. One can thus exploit results from matrix algebra and group rings as needed.

For every $u \in R G$, its $R G$-matrix $\sigma(u)$ is denoted by the corresponding capital letter $U$.

The next useful result, shown also in [7], is that in a group algebra, every element must be a unit or zero divisor, and that there is a method to determine which.

TheOrem 2.3. Let $R$ be a field. A non-zero $u \in R G$ is a zero divisor if and only if $\operatorname{det}(\sigma(u))=0$, and otherwise a unit.

Thus, when $R$ is a field, an element $u \in R G$ is a zero-divisor if and only if $\operatorname{rank} U<n$ and is a unit if and only if $\operatorname{rank} U=n$. Additionally, it can easily be shown that a finite ring with identity contains only zero-divisors and units.

The isomorphism between group rings and the $R G$-matrices allows the generator and check matrices for the group ring codes, to be defined in Section 3, to be immediately derived from the group ring description.

Many of the results stated here hold for infinite groups with corresponding infinite $R G$-matrices.

2.1.1. Examples of $R G$-matrices. In the cyclic group ring case the matrices are the circulant matrices [3]. All cyclic codes can be generated from singular circulant matrices.

The $R G$-matrix types which turn up as isomorphic to certain group rings include Toeplitz-type matrices, Walsh-Toeplitz matrices and circulant matrices, Toeplitz combined with Hankel-type matrices and block-type circulant matrices.

In the general finite abelian group case, $R G$ is isomorphic to certain block circulant matrices that, when $R$ is commutative, commute and are normal. In the case of an elementary abelian 2-group of rank $m$ and order $2^{m}$, where the matrix size is $2^{m} \times 2^{m}$, the $R G$-matrices are the Walsh-Toeblitz matrices over $R$. In the case of the dihedral group the $R G$-matrices are of the form $\left(\begin{array}{ll}A & B \\ B & A\end{array}\right)$ where $A$ is a (general) circulant matrix and $B$ is a reverse circulant matrix.

Further details on these examples of $R G$-matrices appear in [7].

\footnotetext{
${ }^{2}$ Strictly speaking we have defined a left zero-divisor but in all situations here when an element is a right zero-divisor it will also be a left zero-divisor.

${ }^{3}$ In many situations $u v=1$ automatically implies $v u=1$.
} 
2.2. Element properties. Many concepts and properties of matrices turn out to have useful equivalents in the group ring context. These are inherent to the group ring itself, existing independent of any group listing chosen to establish the isomorphism, while maintaining consistency with their equivalent matrix evocation.

We first define the transpose of a group ring element.

Definition 2.4. The transpose of an element $u=\sum_{g \in G} \alpha_{g} g$ in $R G$ is $u^{T}=\sum_{g \in G} \alpha_{g} g^{-1}$, or equivalently, $u^{T}=\sum_{g \in G} \alpha_{g^{-1}} g$.

This is consistent with the matrix definition of transpose. For a given listing $G=\left\{g_{1}, \ldots, g_{n}\right\}$, let $U$ be the $R G$-matrix of $u$. The entry $(i, j)$ of the $R G$-matrix of $u^{\mathrm{T}}$ is $\alpha_{\left(g_{i}^{-1} g_{j}\right)^{-1}}=\alpha_{g_{j}^{-1} g_{i}}$, so $U^{\mathrm{T}}$ is the $R G$-matrix of $u^{\mathrm{T}}$.

The transpose $u^{T}$ has also been called the canonical antiautomorphism of $u[15]$, denoted $\bar{u}$. When dealing with the cyclic groups, it has been referred to as a transpose [9], and it is associated with the reciprocal polynomial.

DeFInITION 2.5. We say that $u \in R G$ is symmetric if and only if $u^{T}=u$.

Clearly, the definition is consistent as $u$ is symmetric if and only if $U$ is a symmetric matrix.

2.3. Some Notation. We shall denote $W u=\{x u: x \in W\}$ and $u W=\{u x: x \in W\}$.

The notation $\underline{x}$ is used to indicate that $\underline{x}$ is a vector as opposed to an element of the group ring. For $\underline{x}=\left(\alpha_{1}, \alpha_{2}, \ldots, \alpha_{n}\right) \in R^{n}$, the mapping $\zeta(\underline{x})=\sum_{i=1}^{n} \alpha_{i} g_{i}=x$ is an element in $R G$ according to a given listing of $G \cdot \zeta^{-1}(x)$ denotes the inverse map.

3. Codes from group ring encodings. Let $R G$ be the group ring of the group $G$ over the ring $R$. A listing of the elements of $G$ is given by $G=\left\{g_{1}, g_{2}, \ldots, g_{n}\right\}$. Suppose $W$ is a submodule of $R G$, and $u \in R G$ is given.

Definition 3.1. Let $x \in W$. A group ring encoding is a mapping $f: W \rightarrow R G$, such that $f(x)=x u$ or $f(x)=u x$. In the latter case, $f$ is a left group ring encoding. In the former, it is a right group ring encoding.

A code $\mathcal{C}$ derived from a group ring encoding is then the image of a group ring encoding, i.e. for a given $u \in R G, \mathcal{C}=\{u x: x \in W\}$ or $\mathcal{C}=\{x u: x \in W\}$.

Multiplication need not necessarily commute in a group ring. Allowing non-commutative groups enables the construction of non-commutative codes.

Definition 3.2. If $x u=u x$ for all $x$ then the code $\{x u: x \in W\}$ is said to be commutative; and otherwise non-commutative.

When $u$ is a zero-divisor, it generates a zero-divisor code and when it is a unit, it generates a unit-derived code.

There is no restriction on the ring $R$. It can be a field, but the techniques we describe are more general, and enable codes over other rings such as the integers $\mathbb{Z}$, rings of matrices or others.

In practice, the submodule $W$ has dimension $r<n$. It can have the basis $\left\{g_{1}, g_{2}, \ldots, g_{r}\right\}$. Other submodules also turn out to be useful, e.g. as generated by $\left\{g_{k_{1}}, g_{k_{2}}, \ldots, g_{k_{t}}\right\}$ with $1 \leq t<n$ where $\left\{k_{1}, k_{2}, \ldots, k_{t}\right\}$ is a subset of $\{1,2, \ldots, n\}$.

For unit-derived codes, there is complete freedom in the choice of $W$ (and hence $r$ ). Zero-divisor codes, as we show in Section 4, have restrictions placed on what $W$ can be in order for a one-to-one map from the code back to $W$ to exist.

When $R G$ is finite and has an identity, only zero-divisors and units are contained in $R G$. This is also true when $R$ is a field by Theorem 2.3. We note, in passing, that in other cases it is possible to generate codes from group ring encodings which are neither zero-divisors nor units, producing a so-called non-zero divisor code. Investigation of the properties of such codes is under investigation.

4. Codes from zero-divisors. We now concentrate on constructing codes from zero-divisors in a given group ring $R G$.

Assume $G$ is of order $n$ with listing $\left\{g_{1}, g_{2}, \ldots, g_{n}\right\}$. The code will be of length $n$ and its dimension will depend on the choice of the submodule $W$. The presentation will first deal with details from the group ring, and then incorporate the matrix algebra relationship. 
Let $u$ be a zero-divisor in $R G$, i.e. $u v=0$ for some non-zero $v \in R G$. Let $W$ be a submodule of $R G$ with basis of group elements $S \subseteq G$.

As defined in Section 3, a resultant zero-divisor code is $\mathcal{C}=\{u x: x \in W\}=u W$ or $\mathcal{C}=\{x u: x \in W\}=$ $W u$. The code is thus constructed from a zero-divisor $u$, a submodule $W$ and, for $R G$ non-commutative, a choice over left or right encoding. We shall describe the case of right-encoding, that is $\mathcal{C}=W u$. The left-encoding case is similar but here we need to consider $v u=0$.

We say that $u$ is a generator element of the code $\mathcal{C}=W u$ relative to the submodule $W$. It is of course possible that $\mathcal{C}$ has another generator element and indeed may also be defined in terms of a different submodule $W$.

The case when $W u=R G u$ is the particular traditional case where the code is a left ideal - see Section 7 for a more complete discussion on this. This is the case where $\operatorname{rank} U$ has the same rank or dimension as $W u$.

When $u$ is a zero-divisor then there is an element $v \neq 0$ with $u v=0$ and thus $y \in \mathcal{C}$ satisfies $y v=0$. It may happen that such an element $v$ which will also determine the code.

Definition 4.1. $v \in R G$ is said to be a (left) check element for a zero-divisor code $\mathcal{C}$ when $y \in \mathcal{C}$ if and only if vy $=0$. We can then write $\mathcal{C}=\{y \in R G: v y=0\}$.

We shall show that given a zero-divisor $u$ and the code $\mathcal{C}$ there is a set $v_{1}, v_{2}, \ldots, v_{t}$ of elements in $R G$ such that $y \in \mathcal{C}$ if and only if $y v_{i}=0$ for $1 \leq i \leq t$.

Zero-divisor codes with a single check element are particularly useful and exist in many cases.

We have defined codes in $R G$ as generated by a zero-divisor and relative to a submodule $W$. We note that in addition to using a zero-divisor as a generator, codes can also be constructed by using a zero-divisor instead directly as a check element, regardless of whether it has a single generating element or not.

Definition 4.2. Suppose $T$ is a submodule of $R G$. Define $T_{v}=\{x \in T \mid x v=0\}$ and say $T_{v}$ is the check zero-divisor code relative to $T$.

Note that $T_{v}$ is a submodule of $R G$ and in the case where $T=R G$ we have that $T_{v}$ is actually a left ideal. It only makes sense to consider the case where $v$ is a zero-divisor in which case $T_{v} \not \supset$. In some cases this code will have a single generator matrix but in any case it will be possible to describe a set of generator elements,

4.1. Modules. Here we are now restricting our attention to the case when $R$ is a field. Some of the results hold over integral domains and also for rings in general but we do not deal with these complications here.

(In the unit-derived codes, Section 5, we do not have the same restrictions.).

Definition 4.3. A set of group ring elements $T \subset R G$ is linearly independent if, for $\alpha_{x} \in R$, $\sum_{x \in T} \alpha_{x} x=0$ only when $\alpha_{x}=0$ for all $x \in T$. Otherwise, $T$ is linearly dependent. We define $\operatorname{rank}(T)$ to be the maximum number of linearly independent elements of $T$. Thus $\operatorname{rank}(T)=|T|$ if and only if $T$ is linearly independent.

Note that a zero-divisor code $\mathcal{C}=W u$, where $W$ is generated by $S$, is the submodule of $R G$ consisting of all elements of the form $\sum_{g \in S} \alpha_{g} g u$. The dimension of this submodule is thus $\operatorname{rank}(S u)$.

If $S u$ is linearly dependent then there exist a subset $S^{\prime} u$ of $S u$ which is linearly independent and generates the same module as $S u .{ }^{4}$ Let $W^{\prime}$ to be the submodue of $W$ generated by $S^{\prime}$ and then the code $\mathcal{C}=W u=W^{\prime} u$, and $S^{\prime} u$ is linearly independent.

The maximum dimension a code for a given zero-divisor $u$ is $r=\operatorname{rank}(G u)$.

The zero-divisor codes are thus $(n, k)$ codes for where $k=\operatorname{rank}(S u)$ and $k \leq r=\operatorname{rank}(G u)$. As pointed out for a given $u$ and a given $W$ it is always possible to find a submodule $W^{\prime}$ of $W$ which may be $W$ itself such that $W^{\prime}$ is generated by $S^{\prime}$ with $S^{\prime} u$ linearly independent and $W u=W^{\prime} u$. One way of finding $S^{\prime}$ inside $S$ is explained below using the matrix $U$ of $u$ and finding an appropriate basis for the matrix consisting of the relevant rows of $U$ corresponding to the elements of $S u$.

A way of finding an $(n, t)$ zero-divisor code is to find $t$ linearly independent rows $i_{1}, i_{2}, \ldots, i_{t}$ of $U$. Then $S=g_{i_{1}}, g_{i_{r} 2}, \ldots, g_{i_{t}}$ is such that $S u$ is linearly independent and generates an $(n, t)$ code. The case $t=\operatorname{rank}(U)$ is the code $R G u$ and can be obtained from considering (any) $t=\operatorname{rank}(U)$ linearly independent

\footnotetext{
${ }^{4}$ It is here we require that $R$ be a field.
} 
rows of $U$. The codes with $t<\operatorname{rank}(S u)$ can be considered as 'shortened' codes. Their generator and check matrices are easly obtained by methods described below.

Notice that to say $S u$ linearly independent is equivalent to saying that $W$ contains no zero-divisors of $u$.

For $G$ the cyclic group $C_{n}=\left\{1, g, g^{2}, \ldots, g^{n-1}\right\}$, let $r$ be the first value such that $\left\{u, g u, g^{2} u, \ldots, g^{r} u\right\}$ is linearly dependent. Then $r$ is the $\operatorname{rank}(u)$, and any subset of $S=\left\{1, g, g^{2}, \ldots, g^{r-1}\right\}$ can be chosen to generate $W$. The proof of this is straighforward and is given in Section 7.4 below. Shortened cyclic codes are obtained by choosing a subset of this set $S$.

4.2. Equivalent Codes in $R^{n}$. The established relationship between the group ring and matrices in Section 2.1 enables us to express the code in terms of matrices, and to derive generator and check matrices for an equivalent code. More precisely, we shall define two equivalent codes in the module $R^{n}$, the second of which has generator/check matrices of the usual form.

Let $U$ be the $R G$-matrix of $u$, and $W$ be a submodule with basis $S=\left\{g_{i_{1}}, g_{i_{2}}, \ldots, g_{i_{r}}\right\}$ such that $S u$ is linearly independent. As previously stated, $\mathcal{C}=W u$ is a code defined on $R G$. A $(n, r)$ code $\mathcal{E}$ can be defined from $R^{r}$ to $R^{n}$ as follows. Let $\underline{w}=\left(\alpha_{1}, \alpha_{2}, \ldots, \alpha_{r}\right) \in R^{r}$ be the vector to be encoded. Using the basis $S$, we write $\underline{w}$ as $\underline{x} \in R^{n}$ with $\alpha_{j}$ in position $i_{j}$ for $1 \leq i \leq r$ and zero everywhere else.

$\underline{x}$ can then be mapped to an element in $W$ by $x=\zeta(\underline{x})=\sum_{j=1}^{r} \alpha_{j} g_{i_{j}}$ and a codeword $x u \in \mathcal{C}$ equated with a codeword in $\mathcal{E}$ given by $\zeta^{-1}(x u)=\underline{x} U$.

In summary, we obtain the $(n, r)$ code $\mathcal{E}=\left\{\underline{x} U: \underline{x} \in R^{r}\right\}$ in $R^{n}$ equivalent to $\mathcal{C}$. Considering codewords as $\underline{x} U \in \mathcal{E}$ where $\underline{x} \in R^{n}$ as described proves, as we will show, convenient for analysis purposes.

For any $n \times n$ matrix $A$ let $\underline{a}_{1}, \underline{a}_{2}, \ldots, \underline{a}_{n}$ denote the rows of $A$ in order.

Generator for matrix-generated code. We can derive a generator matrix $A$ for a code from $R^{r}$ to $R^{n}$ called the matrix-generated code, and given by $\mathcal{D}=\left\{\underline{x} A: x \in R^{r}\right\}$. It is equivalent to codes $\mathcal{C}$ and $\mathcal{E}$.

The codewords in $\mathcal{E}$ consist of linear combinations of the rows $\underline{u}_{i_{1}}, \underline{u}_{i_{2}}, \ldots, \underline{u}_{i_{r}}$ of $U$. Let $A$ be the $r \times n$ matrix consisting of the $i_{1}, \ldots, i_{r}$ rows of $U$. We will show in Lemma 4.6 that the rows of $A$ are linearly independent if and only if $S u$ is.

4.2.1. Linear independence. We now tie up the relationship between linear (in)dependent rows of the $R G$-matrix $U$ and the linear (in)dependence of the set $S u$.

Suppose $S=\left\{g_{i_{1}}, g_{i_{2}}, \ldots, g_{i_{r}}\right\}$ inside $G=\left\{g_{i}, g_{2}, \ldots, g_{n}\right\}$ and that $U$ is obtained from this listing of $G$. Specifically, it is established that the rows $\left\{\underline{u}_{i_{1}}, \underline{u}_{i_{2}}, \ldots, \underline{u}_{i_{r}}\right\}$ of $U$ are linearly independent if and only if $S u$ are.

TheOREM 4.4. Suppose $U$ has rank $t$. Let $S \subset G$ be a set of group elements such that $|S|=t+1$. Then Su is linearly dependent.

Proof. Let the rows of $U$ be $\underline{u}_{1}, \underline{u}_{2}, \ldots, \underline{u}_{n}$ in order.

Suppose $S u=\left\{g_{j_{1}} u, g_{j_{2}} u, \ldots, g_{j_{t+1}} u\right\}$. Any $t+1$ rows of $U$ are dependent so there exists $\alpha_{j_{1}}, \alpha_{j_{2}}, \ldots, \alpha_{j_{t+1}}$ not all zero such that $\sum_{k=1}^{t+1} \alpha_{j_{k}} \underline{u}_{j_{k}}=0_{1 \times n}$. Let $A$ be the $R G$-matrix with first row having $\alpha_{j_{k}}$ in the $j_{k}^{t h}$ position for $1 \leq k \leq t+1$ and zeros elsewhere.

Then $A$ is the $R G$ matrix corresponding to the group ring element $a=\alpha_{j_{1}} g_{j_{1}}+\alpha_{j_{2}} g_{j_{2}}+\ldots+\alpha_{j_{t+1}} g_{i_{t+1}}$. Also $A U$ is an $R G$-matrix whose first row consists of zeros and hence $A U=0_{n \times n}$. Thus $a u=0$ and therefore $\left\{g_{i_{1}} u, g_{i_{2}} u, \ldots, g_{i_{r+1}} u\right\}$ is linearly dependent as required.

Then from the last theorem it follows that we may take $S$ to have $r$ elements such $r \leq \operatorname{rank} U$. For if $r>\operatorname{rank} U$ then $S u$ is generated by $r$ elements $S^{\prime} u$ (where $S^{\prime} \subset S$ ), and the code is given by $\mathcal{C}=W^{\prime} u$ where $W^{\prime}$ is the module generated by $S^{\prime}$.

Alternatively, assuming $U$ has rank $\geq r$, one can choose or find $r$ linearly independent rows $\underline{u}_{i_{1}}, \underline{u}_{i_{2}}, \ldots, \underline{u}_{i_{r}}$ of $U$ and then construct $S$ by reference to these, let $S=\left\{g_{i_{1}}, g_{i_{2}}, \ldots, g_{i_{r}}\right\}$ and then $S u$ is linearly independent.

Define $G_{j}$ to be the $R G$-matrix corresponding to the group element $g_{j} \in G$ - this is consistent with the notation for the $R G$-matrix corresponding to $g_{j}$. Then $G_{j}$ is the matrix whose first row has a 1 in the $j^{\text {th }}$ position and zeros elsewhere. It is then clear that $G_{j} U$ is the $R G$-matrix with first row $\underline{u}_{j}$. 
Lemma 4.5. Suppose $\underline{u}_{1}, \underline{u}_{2}, \ldots, \underline{u}_{s}$ are the first rows (or first columns) of the $R G$-matrices $U_{1}, U_{2}, \ldots, U_{s}$ respectively. Then $\alpha_{1} \underline{u}_{1}+\alpha_{2} \underline{u}_{2}+\ldots+\alpha_{s} \underline{u}_{s}=0$ if and only if $\alpha_{1} U_{1}+\alpha_{2} U_{2}+\ldots+\alpha_{s} U_{s}=0$.

Proof. Suppose $\alpha_{1} \underline{u}_{1}+\alpha_{2} \underline{u}_{2}+\ldots+\alpha_{s} \underline{u}_{s}=0$. Let $U=\alpha_{1} U_{1}+\alpha_{2} U_{2}+\ldots+\alpha_{s} U_{s}$. Then $U$ is an $R G$-matrix whose first row consists of zeros and hence $U=0$.

On the other hand it is clear that if $\alpha_{1} U_{1}+\alpha_{2} U_{2}+\ldots+\alpha_{s} U_{s}=0$ then $\alpha_{1} \underline{u}_{1}+\alpha_{2} \underline{u}_{2}+\ldots+\alpha_{s} \underline{u}_{s}=0$.

LEMMA 4.6. $\left\{g_{i_{1}} u, g_{i_{2}} u, \ldots, g_{i_{r}} u\right\}$ is linearly independent if and only if $\left\{\underline{u}_{i_{1}}, \underline{u}_{i_{2}}, \ldots, \underline{u}_{i_{r}}\right\}$ is linearly independent.

Proof. Follows immediately from Lemma 4.5.

Suppose then $\operatorname{rank} U=r$ and that $\left\{\underline{u}_{i_{1}}, \underline{u}_{i_{2}}, \ldots, \underline{u}_{i_{r}}\right\}$ is linearly independent. Then by Lemma 4.6, $S u$ is linearly independent. It is also clear in this situation that $\mathcal{C}=R G u$, the right ideal generated by $u$.

4.3. Check elements and matrices. Throughout this section(4.3), the code under question is $(n, r)$ where $r=\operatorname{rank} U$. In Section 4.4, we describe how to obtain check conditions for $(n, k)$ codes where $k<\operatorname{rank} U$.

Clearly $c v=0$ for any codeword $c$. The most convenient situation is when the code $\mathcal{C}$ (and thus for codes $\mathcal{D}, \mathcal{E}$ ) has a (single) check element, i.e. that $y \in C$ is a codeword if and only if $y v=0$. Equivalently, $V$ checks $\mathcal{E}$ provided $\underline{y} \in \mathcal{E}$ if and only if $\underline{y} V=0$ if and only if $Y V=0$, where $\underline{y}$ is the first row of $Y$. We now examine these requirements for a check element.

4.3.1. Check elements. Definition 4.7. For a zero-divisor $u$ with $\operatorname{rank} U=r$, say $u$ is a principal zero-divisor if and only if there exists a $v \in R G$ such that $u v=0$ and $\operatorname{rank} V=n-r$.

This is the situation for example when $R G$ is a principal ideal domain as when $G$ is a cyclic group - see Section 7 for a discussion and proof of this in the cyclic group ring case.

It is also possible in other cases that for a given zero-divisor $u$ there is a $v$ with $u v=0$ and $\operatorname{rank} U+$ $\operatorname{rank} V=n$; for example if $u^{2}=0$ or $u u^{T}=0$ and $\operatorname{rank} U=\frac{n}{2}$, in which case $\operatorname{rank} U^{T}=\frac{n}{2}$ also.

Suppose that $u v=0$ and $\operatorname{rank} V=n-r$. Then $y$ is a codeword if and only if $y v=0$ if and only if $Y V=0$. This is not immediately obvious and depends on the fact that $U$ and $V$ are $R G$-matrices; the proof, in stages, is shown below.

Lemma 4.8. Let $\underline{y}$ be the first row of an $R G$-matrix $Y$. Suppose also $V$ is an $R G$-matrix. Then $Y V=0$ if and only if $\underline{y} V=0$.

Proof. Suppose $\underline{y} V=0$. Then $Y V$ is an $R G$-matrix with first row consisting of zeros. Hence $Y V=0$. On the other hand if $Y V=0$ then clearly $y V=0$.

TheOREM 4.9. Let $\mathcal{C}=\{x u: x \in W\}$ where $W$ is generated by $S$ such that $S u$ is linearly independent and $|S|=\operatorname{rank} U=r$. Suppose further that $u v=0$ in the group ring $R G$ so that $\operatorname{rank} V=n-r$. Then $y$ is a codeword if and only if $y v=0$.

Proof. (We need to show that $y v=0$ if and only $y=\alpha u$ for some $\alpha \in W$.) If $y$ is a codeword then $y=x u$ for some $x \in W$ and hence $y v=0$.

Suppose on the other hand $y v=0$. Now $U V=0$ where $U$ has rank $r$ and $V$ has rank $n-r$. The null-space of $V$ is the set of all (row) vectors $\underline{x}$ such that $\underline{x} V=0$. Since $V$ has rank $n-r$, by linear algebra the rank of the null-space of $V$ is $r$. Since $U$ has rank $r$ the rows of $U$ generate the null-space of $V$.

Since also $Y V=0$ the rows of $Y$ are in the null-space of $V$ and hence the rows of $Y$ are linear combinations of the rows of $U$. In particular $\underline{y}=\underline{a} U$ where $\underline{y}$ is the first row of $Y$ and $\underline{a}$ is a $1 \times n$ vector. Let $Q$ be the $R G$-matrix whose first row is $\underline{a} ; R G$ matrices are uniquely defined by their first row. Then $Q U$ is an $R G$-matrix whose first row is $\underline{y}$, the first row of $Y$. Hence $Y=Q U$. From this it follows that $w=q u$ (where $q$ is the group ring element corresponding to the $R G$-matrix $Q$ ). We need to show that $q u \in \mathcal{C}$. Let $q=\sum_{i=1}^{n} \alpha_{i} g_{i}$. Suppose $g_{j}$ occurs in this sum with non-zero coefficient and $g_{j} \notin S$. Then $\left\{g_{i_{1}} u, g_{i_{2}} u, \ldots, g_{i_{r}} u, g_{j} u\right\}$ is linearly dependent by Theorem 4.4, the first $r$ of which is linearly independent. Hence $g_{j} u$ is in $\mathcal{C}$ as required. $\square$

Corollary 4.10. $\mathcal{C}=\{x u: x \in W\}$ has a single check element if and only if $u$ is a principal zero-divisor: $\mathrm{Q}$

Corollary 4.11. $\underline{y} \in \mathcal{E}$ if and only if $\underline{y} V=0$ if and only if $Y V=0$ where $Y$ is the RG-matrix with first row $\underline{y} \cdot \square$ 
4.3.2. General check conditions. Define the null-space of $U$ to be $\operatorname{Ker}(U)=\{\underline{x}: U \underline{x}=0\}$ where $\underline{x}$ is an $n \times 1$ vector. Since $U$ has rank $r$, the dimension of $\operatorname{Ker}(U)$ is $n-r$. Let $\underline{v}_{1}, \underline{v}_{2}, \ldots, \underline{v}_{n-r}$ be a basis for $\operatorname{Ker}(U)$; these $\underline{v}_{i}$ are $n \times 1$ column vectors. Let $V_{i}$ be the $R G$-matrix with first column $\underline{v}_{i}$. Then clearly $U V_{i}=0$ for $1 \leq i \leq n-r$ since $U V_{i}$ is the $R G$-matrix with first row consisting of zeros and hence must be zero. Hence $u v_{i}=0$ where $v_{i}$ is the group ring element corresponding to the $R G$-matrix $V_{i}$.

Note that the null-space of $U$ is easily and quickly obtained using linear operations on the rows of $U$. The basis for the null-space may be read off from the row-reduced echelon form of $U$, which also puts the generator in standard form. This is also very useful in producing a check matrix for the corresponding encoding $R^{r} \rightarrow R^{n}$ - see Theorem 4.14 below.

Thus if $y$ is a codeword then $y v_{i}=0$ for $1 \leq i \leq n-r$. The following theorem may be proved along similar lines to Theorem 4.9. Its proof is omitted.

THEOREM 4.12. Suppose $u$ is a zero-divisor, $\operatorname{rank} U=r$ and $W$ is generated by $S$ with $r$ elements such that $S u$ is linearly independent. Let $v_{i}$ be defined as above. Then $y \in \mathcal{C}$ if and only if $y v_{i}=0$ for all $i=1, \ldots, n-r . \mathrm{Q}$

Corollary 4.13. $Y$ is a codeword if and only if $Y V_{i}=0$

Not all the $v_{i}$ are needed - just enough so that the corresponding matrices $V_{i}$ contain a basis for the null-space. In many cases a particular $V_{i}$ of rank $n-r$ can be found. The check conditions for the code $\mathcal{E}$ follow:

THEOREM 4.14. Let $\hat{V}=\left(v_{1}, v_{2}, \ldots, v_{n-r}, 0,0, \ldots, 0\right)$ be the $n \times n$ matrix with first $n-r$ columns consisting of $v_{i}$ in order and then $r$ columns with zeros. Then $\underline{y} \in \mathcal{E}$ if and only if $\underline{y} \hat{V}=0$.

Proof. Clearly if $\underline{y} \in \mathcal{E}$ then $\underline{y} \hat{V}=0$.

Suppose then $\underline{y} \hat{V}=0$. Define $\operatorname{Ker}(\hat{V})=\left\{t \in R^{n}: t \hat{V}=0\right\}$. Since $\hat{V}$ has dimension $n-r$, the dimension of $\operatorname{Ker}(\hat{V})$ is $r$. Now each row of $U$ is in $\operatorname{Ker}(\hat{V})$ since $U \hat{V}=0$. Since $U$ also has rank $r$ this implies the rows of $U$ generate $\operatorname{Ker}(\hat{V})$. Hence $y$ is a linear combination of the rows of $U$. The rows $u_{i_{1}}, u_{i_{2}}, \ldots, u_{i_{r}}$ are linearly independent and hence are a basis for the row space of $U$ which has dimension $r$. Hence $\underline{y}=\alpha_{1} u_{i_{1}}+\alpha_{2} u_{i_{2}}+\ldots+\alpha_{r} u_{i_{r}}$ and is thus a codeword. $\mathbf{\square}$

4.3.3. Generator from a check element. The argument above may also be used to obtain a generator when we use a zero-divisor $v \in R G$ to act as a check element and produce the code $T_{v}=\{y \in: T y v=0\}$, regardless of whether the code has a single generating element or not.

Take the case $T=R G$. Suppose the resultant $R G$-matrix $V$ has rank $n-r$. Then $n-r$ of the rows of $V$ are linearly independent and the other rows of $V$ are linearly combinations of these. Thus the code may be considered a $(n, r)$ code with check matrix of size $(n-r) \times n$.

Define $\operatorname{Ker}(V)=\{\underline{x}: \underline{x} V=0\}$. Then $\operatorname{Ker}(V)$ has rank $r$. Let $\underline{u}_{1}, \underline{u}_{2}, \ldots, \underline{u}_{r}$ be a basis for $\operatorname{Ker}(V)$. Form the matrix $\hat{U}$ with rows $\underline{u}_{i}$. Then we get the following:

TheOREM 4.15. $\hat{U}$ is a generator matrix of the code $\mathcal{E}$.

Proof. The proof is similar to that of Theorem 4.14.

4.4. Check matrices when the dimension is less than the rank. We now take the case where $W$ is the submodule generated by $S$ such that $|S|=s<r=\operatorname{rank} U$. This generates an $(n, s)$ code.

One way to create a check matrix for $\mathcal{D}$ is to apply standard row operations to obtain a basis for the null-space of the generator $A$. However, it can also be obtained from the $R G$-matrices $U$ and $V$ by adding certain $r-s$ vectors to $V$ as explained below.

Let $V_{n-r}$ denote a submatrix of $V$ consisting of $n-r$ linearly independent columns.

Consider the indexing set $T=\left\{k_{1}, k_{2}, \ldots, k_{s}\right\}\left(1 \leq k_{1}<k_{2}<\ldots<k_{s} \leq n\right)$ which defines $S=$ $\left\{g_{k_{1}}, \ldots, g_{k_{s}}\right\}$. Extend the set $T$ to a set of linearly independent rows $R=\left\{k_{1}, k_{2}, \ldots, k_{s}, w_{1}, \ldots, w_{r-s}\right\}$ of $U$.

Let $U_{r}$ be the matrix formed from $R$ with the rows in order. Then $U_{r}$ has rank $r$ and size $r \times n$. There exists an $n \times r$ matrix $C$ such that $U_{r} C=I_{r}$.

Delete the $k_{1}, k_{2}, \ldots, k_{s}$ columns of $C$ to get an $n \times(r-s)$ matrix, which we call $C_{r-s}$. We now add this $C_{n-r}$ matrix to $V_{n-r}$ to get the matrix $D$. This $D$ then has rank $n-s$ and satisfies $U_{r} D=0$. It follows that $\underline{y} \in \mathcal{D}$ if and only if $D^{\mathrm{T}} \underline{y}^{\mathrm{T}}=0$. 
Thus $D^{\mathrm{T}}$ is a check matrix for $\mathcal{D}$, obtained by adding certain $r-s$ columns from $C$, the right inverse of $U_{r}$, to the matrix $V_{n-r}$.

4.5. Dual and self-check codes. By definition, a dual of a code $\mathcal{C}$ considered as vectors over $R^{n}$ is its orthogonal complement, namely $\mathcal{C}^{\perp}=\left\{v \in R^{n}:\langle v, c\rangle=0, \forall c \in \mathcal{C}\right\}$.

Let $x, y \in R G$. The inner (or dot) product, is given by term-by-term multiplication of the elements in the ring $R$, namely $\langle x, y\rangle=\sum_{g \in G} \alpha_{g} \beta_{g}$ where $x=\sum_{g \in G} \alpha_{g}$ and $y=\sum_{g \in G} \beta_{g}$.

Thus, the dual of a code from a group ring encoding is $\mathcal{C}^{\perp}=\{y \in R G:\langle u x, y\rangle=0, \forall x \in W\}$. We now show that the dual of a zero-divisor code has an easy form.

TheOREm 4.16. Let $u, v \in R G$ such that $u v=0$. Let $U$ and $V$ be the $R G$-matrices of $u$ and $v$ respectively, such that $\operatorname{rank} U=r$ and $\operatorname{rank} V=n-r$. Let $W$ be a submodule over a basis $S \subset G$ of dimension $r$ such that $S u$ is linearly independent and $W^{\perp}$ denote the submodule over basis $G \backslash S$. Then the code $\mathcal{C}=\{x u: x \in W\}$ has dual code $\mathcal{C}^{\perp}=\left\{x v^{T}: x \in W^{\perp}\right\}=\left\{y \in R G: y u^{T}=0\right\}$.

Proof. Note that $v^{\mathrm{T}}$ is a zero-divisor and that $\operatorname{rank} V^{\mathrm{T}}=n-r$ (because $\operatorname{rank} V=n-r$ ), and that $W^{\perp}$ does not contain a zero-divisor of $v^{\mathrm{T}}$. Thus, there is a 1-1 map between $W^{\perp}$ and $\left\{x v^{\mathrm{T}}: x \in W^{\perp}\right\}$. It remains to show it is the dual.

Let $z \neq 0$ be an element in $R G$. We need to prove that $\langle x u, z\rangle=0, \forall x \in W$ if and only if $z=y v^{\mathrm{T}}$ for some $y \in W^{\perp}$.

Suppose $z=y v^{\mathrm{T}}$, and let $x, y \in R G$.

Recall that $\underline{x}=\zeta^{-1}(x), \underline{y}=\zeta^{-1}(y)$ are the vectors in $R^{n}$ corresponding to $x, y$. Then, $\langle x u, z\rangle=$ $\left\langle x u, y v^{\mathrm{T}}\right\rangle=\underline{x} U\left(V^{\mathrm{T}} \underline{y}\right)^{\mathrm{T}}=\underline{x}(U \bar{V}) \underline{y}^{\mathrm{T}}=0$.

Conversely, suppose $\langle x u, z\rangle=0, \forall x \in W$. Without loss of generality, assume $1 \in W$. Then $\langle u, z\rangle=0$ implies $z u^{\mathrm{T}}=0$, and since $u^{\mathrm{T}}$ is the check element for the code generated by $v^{\mathrm{T}}, z=y v^{\mathrm{T}}$ for some $y \in W^{\perp}$. 口

This is consistent with cyclic codes whereby the dual for a code with generator $u$ and check $v$ is usually expressed as having the reciprocal (polynomial) $g^{n-r} v^{\mathrm{T}}$ (where the $\operatorname{deg}(v)=n-r$ ) as generator [2]. Using this as generator with $W$ having basis $\left\{1, g, \ldots, g^{r}\right\}$ yields the same code as generator $v^{\mathrm{T}}$ with submodule $W^{\perp}$.

Of course, for the matrix-generated code $\mathcal{D}$, one may obtain a code equivalent to its dual by interchanging the generator and check matrices; see for example[10].

A condition for self-duality is an easy consequence.

Corollary 4.17. $\mathcal{C}^{\perp}=\mathcal{C}$ if and only if $u u^{T}=0$ and $\operatorname{rank} U=n / 2$. $\square$

A self-dual zero divisor code is thus a code given by $u$ with $u u^{\mathrm{T}}=0$ and $\operatorname{rank} U=n / 2$. We say a code given by $u$ is self-check if $u^{2}=0$, in which case it is equivalently self-dual, as the code and its dual are equivalent.

4.5.1. Examples of zero-divisor self-dual codes. Group rings are a rich source of elements $u$ such that $u^{2}=0$ or $u u^{T}=0$ or both. These will generate self-dual (or self-check) codes when $\operatorname{rank} U=n / 2$. See Section 6.1 for specific examples.

5. Codes from units. In this section, we construct codes from units in group rings. Let $u$ be a unit in the $R G$, where $G$ is of order $n$ and listed $G=\left\{g_{1}, g_{2}, \ldots, g_{n}\right\}$. Let $W$ be a submodule of $R G$ generated (as an $R$-module) by $r$ group elements $S=\left\{g_{k_{1}}, g_{k_{2}}, \ldots, g_{k_{r}}\right\}$ with $r<n$.

As defined in Section 3, the unit-derived code is $\mathcal{C}=\{u x: x \in W\}$ or $\mathcal{C}=\{x u: x \in W\}$. The code is thus constructed from a unit $u$, a submodule $W$ and, when $R G$ does not commute, a choice over left or right encoding. Assume in what follows that the encoding is on the right $(x \mapsto x u)$. The left-encoding case $x \mapsto u x$ is similar, following, with minor adjustments, the same general procedure.

Now $c$ is a codeword (i.e. in $\mathcal{C}$ ) if and only if $c u^{-1} \in W$ i.e. if and only if the coefficients of $G \backslash S$ in $c u^{-1}$ are zero. Notice that multiplying a codeword by the inverse of the unit recovers the original.

A unit-derived code can also be considered a mapping from $R^{r}$ to $R^{n}$. First, map a vector $\underline{x}=$ $\left(\alpha_{1}, \alpha_{2}, \ldots, \alpha_{r}\right) \in R^{r}$ by $\lambda_{W}(\underline{x})=\sum_{i=1}^{r} \alpha_{i} g_{k_{i}}$ to an element $x \in W$. Then a codeword $x u \in \mathcal{C}$ is obtained 
which may be written $x u=\sum_{i=1}^{n} \beta_{i} g_{i}$. This gives an encoding $\underline{x} \mapsto\left(\beta_{1}, \beta_{2}, \ldots, \beta_{n}\right)$ which is a map from $R^{r} \rightarrow R^{n}$.

We also associate each unit-derived code with an equivalent code which we call the matrix-generated code $\mathcal{D}$. This is a code from $R^{r}$ to $R^{n}$ and has an $r \times n$ generator matrix $A$ extracted from the $R G$-matrix $U$, and a check matrix that extracted from $V$. If $A$ is such a generating matrix, then $\mathcal{D}=\left\{\underline{x} A: \underline{x} \in R^{r}\right\}$. The distinction between codes $\mathcal{C}$ and $\mathcal{D}$ is one of convenience. They are equivalent, exhibiting the same properties. This procedure is conceptual and any practical implementation works on producing $\mathcal{D}$ or $\mathcal{C}$ directly, depending on what is desired.

5.1. Generator and Check Matrices. Let us now examine the check and generator matrices that result from a unit-derived code. Suppose $u u^{-1}=1$ in the group ring and let $U, U^{-1}$ respectively be the corresponding $n \times n R G$-matrices.

First, consider $W$ to be the submodule generated by $\left\{g_{1}, g_{2}, \ldots, g_{r}\right\}$ with $r<n$ (i.e. has as basis the first $r$ elements in the chosen listing of $G$ ). We later deal with the case when $W$ has a general basis of group elements. An element in $W$ is thus of the form $x=\sum_{i=1}^{r} \alpha_{i} g_{i}$.

Divide $U=\left(\begin{array}{l}A \\ B\end{array}\right)$ into block matrices where $A$ is $r \times n$ and $B$ is $(n-r) \times n$. Similarly, let $U^{-1}=\left(\begin{array}{ll}C & D\end{array}\right)$ where $C$ is $n \times r$ and $D$ is $n \times(n-r)$.

Now $A D=0$ as $U U^{-1}=I$. It is easy to see that $A$ is a generator matrix for the matrix-generated code. We now show that $D^{\mathrm{T}}$ is a check matrix.

TheOREm 5.1. Let $\underline{y} \in R^{n}$ and $\mathcal{D}=\left\{\underline{x} A: \underline{x} \in R^{r}\right\}$. Then $\underline{y} \in \mathcal{D}$ if and only if $\underline{y} D=0$.

Proof. If $\underline{y}=x A$ for some $\underline{x} \in R^{r}$, then clearly $\underline{y} D=0$. If, on the other hand, $\underline{y} D=0$,

$$
\underline{y}=\underline{y} U^{-1} U=\underline{y}\left(\begin{array}{ll}
C & D
\end{array}\right)\left(\begin{array}{l}
A \\
B
\end{array}\right)=\left(\begin{array}{ll}
\underline{y} C & \underline{y} D
\end{array}\right)\left(\begin{array}{ll}
A & B
\end{array}\right)=\left(\begin{array}{ll}
\underline{y} C & 0
\end{array}\right)\left(\begin{array}{l}
A \\
B
\end{array}\right)=\underline{y} C A .
$$

Now $\underline{y} C$ is in $R^{r}$ and $\underline{y}=\underline{y} U^{-1} U=\underline{x} A$ for some $\underline{x} \in R^{r}$ as required. $\square$

Thus $D^{\mathrm{T}}$ is a check matrix for the matrix-generated code $\mathcal{D}: \underline{y}$ is a codeword if and only if $D^{\mathrm{T}} \underline{y}^{\mathrm{T}}=0$ if and only if $y D=0$. The $r \times n$ generator matrix $A$ and $(n-r) \times n$ check $D^{\mathrm{T}}$ produced from this unit and submodule have full allowable rank, $r$ and $n-r$ respectively.

Units in group rings result in non-singular matrices, enabling the construction of codes from units. Any non-singular matrix could also produce a code by the above arguments, although of course one could not exploit the underlying algebraic structure of a group ring.

When $W$ is generated by a general basis $S=\left\{g_{k_{1}}, g_{k_{2}}, \ldots, g_{k_{r}}\right\}$, the generator and check matrices are obtained by 'extracting' from and 'adding' to certain rows and columns from $U$ and $U^{-1}$. A generator matrix results from the $r \times n$ matrix consisting of the $k_{1}, k_{2}, \ldots, k_{r}$ rows of $U$. Additionally, let $D$ be the $(n-r) \times n$ matrix obtained by deleting the $k_{1}, k_{2}, \ldots, k_{r}$ columns of $V$. Then $D^{\mathrm{T}}$ is a check matrix.

5.2. Constructing unit-derived codes. The generator and check matrices for the matrix-generated code $\mathcal{D}$ are immediate from the construction. However, working with the unit-derived code $\mathcal{C}$ itself can be advantageous. For example, using the group ring check conditions directly may be the best method for decoding.

In summary, unit-derived code of length $n$ and dimension $r$ can be constructed quite freely as follows. Choose a group $G$ of order $n$ and a ring $R$ over which the code will be defined. Typically, $R$ is a field but this is not a requirement; codes over the integers, rings of matrices or other rings are also useful.

Find a unit $u$ in the group ring $R G$ and its inverse $u^{-1}$. As previously mentioned, if $R$ is a field or $R G$ of finite order,

every element in $R G$ is either a zero-divisor or a unit. When $R$ is a field, there is a straightforward algorithm to determine which. Generation of units is therefore not difficult.

Any basis for a submodule $W$ consisting of $r$ group elements will generate a code, e.g. the first $r$ elements $\left\{g_{1}, g_{2}, \ldots, g_{r}\right\}$ according to a listing of $G$. 
It can proves advantageous to choose another, appropriate, basis, to say increase the minimum distance of the code or optimise some other criteria. This freedom of basis nicely leads to the concept of an optimal one for a given unit $u \in R G$ and dimension $r$ - a so-called best-basis:

$$
\underset{S \subset G,|S|=r}{\arg \max } \min _{x \in W(S)} \operatorname{wt}(x u)
$$

where $W(S)$ denotes the submodule generated by $S$ and wt $(y)$ the number of nonzero coefficients of $y$.

This flexibility in choice of $r$ and $W$ and the full ranks obtained are major advantages of a unit-derived code over one derived from a zero-divisor.

5.3. Derivation of Units. Group rings are a rich source of units. Units exist and are known in $R G$, where $R$ can be any ring and not just a field, and from these, codes of different types and makes can be constructed. Once a unit is known there is still a choice on the submodule/dimension for the code and codes of different dimensions may be obtained from a particular unit.

To fully describe a unit-derived code in terms of generator and check conditions we need a unit and its inverse. The inverse may be known from the algebra; general formulae for certain units, and their inverses, in group rings are known. Please consult [12] and the references therein. In the cases of group rings over cyclic groups it is worth noting that the Euclidean Algorithm, which is extremely fast, may be used to obtain an inverse as then $R G \cong R[x] /\left\langle x^{n}-1\right\rangle$. A variation of the Euclidean Algorithm may also be used to find inverses in $R G$ when $G$ is a dihedral group.

Computer Algebra packages such as GAP, MAGMA which are particularly useful for handling groups, may in addition be used to find units in group rings; for example, there exist function such as DihedralGroup() and DirectProduct() which return dihedral groups and direct product of groups respectively.

The combination of units in a group ring is also a unit. This can be exploited to produce new units which are not of the same form as the originals and from which new codes can be derived. Thus, for example, bicyclic units could be combined with alternating units, Hoeschmann units etc. to give new types of units.

5.4. Dual and Orthogonal Codes. Recall from Section 4.5 that the dual of a code from a group ring encoding is $\mathcal{C}^{\perp}=\{y \in R G:\langle u x, y\rangle=0, \forall x \in W\}$ and the concept of the transpose of a group ring element (Definition 2.4). We now show that the dual of a unit-derived code can be generated from $\left(u^{-1}\right)^{\mathrm{T}}$.

THEOREM 5.2. Let $W$ be a submodule with basis of group elements $S \subset G$ and $W^{\perp}$ be the submodule with basis $G \backslash S$. Let $u \in R G$ be a unit such that $u u^{-1}=1$. Then the dual code of $\mathcal{C}=\{x u: x \in W\}$ is $\mathcal{C}^{\perp}=\left\{x\left(u^{-1}\right)^{T}: x \in W^{\perp}\right\}$.

Proof. Let $z \neq 0$ be an element in $R G$. We need to show that $\langle x u, z\rangle=0, \forall x \in W$ if and only if $z u^{\mathrm{T}} \in W^{\perp}$.

Note that $\left\langle x u, y\left(u^{-1}\right)^{\mathrm{T}}\right\rangle=\langle x, y\rangle$. Thus, if $z u^{\mathrm{T}} \in W^{\perp}$, then, for all $x \in W,\langle x u, z\rangle=\left\langle x, z u^{\mathrm{T}}\right\rangle=0$.

Conversely, if $z u^{\mathrm{T}} \in W$, pick a $g \in S$ that has a non-zero coefficient $\gamma$ in $z u^{\mathrm{T}}$. Then, $\langle g u, z\rangle=\left\langle g, z u^{\mathrm{T}}\right\rangle=$ $\gamma \neq 0$. $\mathrm{P}$

Strict equivalence of a unit-derived code and its dual, whereby $\mathcal{C}=\mathcal{C}^{\perp}$, requires that for all $x \in W$, $x u u^{\mathrm{T}} \in W^{\perp}$, which imposes an impractical restriction. However, it is natural to say that a unit-derived code is self-dual if $\mathcal{C}$ and $\mathcal{C}^{\perp}$ are equivalent codes, or equivalently, that the resultant matrix-generated codes $\mathcal{D}$ and $\mathcal{D}^{\perp}$ are equal.

Definition 5.3. An unit $u \in R G$ is orthogonal if and only if its inverse is $u^{T}$ (i.e. $u u^{T}=1$ ).

It is easy to see that the $R G$-matrix from an orthogonal unit $u$ is an orthogonal matrix. From the above, it can also be seen that an orthogonal unit combined with a submodule of dimension $n / 2$ generates a self-dual unit-derived code.

6. Examples codes. In this section, we explore some illustrative constructions of codes from group ring encodings. The examples below include codes in general abelian groups and also in non-abelian groups.

6.1. Self-dual codes. Some self-dual codes in $R G$ can be formed as follows. Suppose $|G|=n=2 m$ and $G=\left\{g_{1}, g_{2}, \ldots, g_{n}\right\}$. Let $u \in R G$ satisfy:

1. $u^{2}=0$

2. $u=u^{\mathrm{T}}$ so that $u u^{\mathrm{T}}=0$.

3. $u$ and its corresponding matrix $U$ have rank $m$. 
Then $u$ generates a self-dual code. Here's a specific example. Let $G=C_{2} \times C_{4}$ where $C_{4}$ is generated by $a$ and $C_{2}$ is generated by $h$. Form the group ring $\mathbb{Z}_{2} G$.

Consider $u=1+h\left(a+a^{2}+a^{3}\right)$. Then $u^{2}=1+h^{2}\left(a^{2}+a^{4}+a^{6}\right)=1+a^{2}+1+a^{2}=0$. Thus rank $u \leq 4$. The $R G$-matrix of $u$ is $U=\left(\begin{array}{cc}I & B \\ B & I\end{array}\right)$ from which it follows that rank $u=4$. By algebraic techniques on the group ring it can be shown that the distance is 4 . We thus get a $(8,4,4)$ self-dual code - this must then be the extended Hamming self-dual code.

Extending this by considering $G=C_{4}^{n} \times C_{2}$ or other direct products is the subject of further work and produces an infinite series of self-dual codes with increasing distance.

6.1.1. Example of unit-derived codes. If $u^{2}=0$ then $(1+u)^{2}=1$ over $\mathbb{Z}_{2}$. Consider $u_{i}=$ $g^{i}+g^{n-i}+g^{n+i}+g^{2 n-i}$ in $\mathbb{Z}_{2} C_{2 n}$, with $C_{2 n}$ generated by $g$. Then $u_{i}^{2}=u_{i} u_{i}^{\mathrm{T}}=0$ and thus any combination, $u$ say, of the $u_{i}$ satisfies $u^{2}=u u^{\mathrm{T}}=0$. Consequently $a=1+u$ satisfies $a^{2}=a a^{\mathrm{T}}=1$, and gives a series of orthogonal units. There is no problem with the rank as we are dealing with unit-derived codes.

A specific example of this is as follows: In $\mathbb{Z}_{2} C_{14}, u=1+g^{2}+g^{5}+g^{9}+g^{12}$ satisfies $u^{2}=u u^{T}=1$. The code has distance $d=4$, and thus we get a $(14,7,4)$ code, with the best possible distance for a $(14,7)$ binary code.

6.2. Dihedral codes. The first natural series of non-abelian groups are the dihedral groups.

Codes from group ring encodings from the dihedral group appear to offer great potential. There has been prior investigation into codes from ideals in the dihedral group algebra as early as 1969[8], and a more recent result [1], which showed that there exist a random ideal in $Z_{2} D_{2 n}$ for infinitely many $n$, such that the resultant code of rate $1 / 2$ is "good".

The focus on using encodings directly and the use of matrix algebra $R G$-matrix structure is, to the best of our knowledge, a new approach.

The dihedral group $D_{2 n}$ of order $2 n$ is given $D_{2 n}=\left\langle a, b: a^{2}, b^{n}, a b=b^{-1} a\right\rangle$. There are a number of possible listings of the elements of $D_{2 n}$, of which $D_{2 n}=\left\{1, b, b^{2}, \ldots, b^{n-1}, a, a b, a b^{2}, \ldots, a b^{n-1}\right\}$ proves most convenient.

An element $u \in R D_{2 n}$ can be written

$$
u=\sum_{i=0}^{n-1} \alpha_{i} b^{i}+\sum_{i=0}^{n-1} \beta_{i} a b^{i} .
$$

The associated $R D_{2 n}$-matrix $W$ is then,

$$
U=\left(\begin{array}{ccccc|ccccc}
\alpha_{0} & \alpha_{1} & \alpha_{2} & \ldots & \alpha_{n-1} & \beta_{0} & \beta_{1} & \beta_{2} & \ldots & \beta_{n-1} \\
\alpha_{n-1} & \alpha_{0} & \alpha_{1} & \ldots & \alpha_{n-2} & \beta_{1} & \beta_{2} & \beta_{3} & \ldots & \beta_{0} \\
\vdots & \vdots & \vdots & \vdots & \vdots & \vdots & \vdots & \vdots & \vdots & \vdots \\
\alpha_{1} & \alpha_{2} & \alpha_{3} & \ldots & \alpha_{0} & \beta_{n-1} & \beta_{0} & \beta_{1} & \ldots & \beta_{n-2} \\
\hline \beta_{0} & \beta_{1} & \beta_{2} & \ldots & \beta_{n-1} & \alpha_{0} & \alpha_{1} & \alpha_{2} & \ldots & \alpha_{n-1} \\
\beta_{1} & \beta_{2} & \beta_{3} & \ldots & \beta_{0} & \alpha_{n-1} & \alpha_{0} & \alpha_{1} & \ldots & \alpha_{n-2} \\
\vdots & \vdots & \vdots & \vdots & \vdots & \vdots & \vdots & \vdots & \vdots & \vdots \\
\beta_{n-1} & \beta_{0} & \beta_{1} & \ldots & \beta_{n-2} & \alpha_{1} & \alpha_{2} & \alpha_{3} & \ldots & \alpha_{0}
\end{array}\right) .
$$

This can be written $U=\left(\begin{array}{ll}A & B \\ B & A\end{array}\right)$, where $A$ is circulant. Now, $B$ is a reverse circulant matrix as each row is a circulant shift to the left of the one previous. Interestingly, in a non-group ring context, reverse circulants have appeared before in codes [5].

A useful method for classification of the units and zero-divisors in such a group ring results. Suppose $R$ is an integral domain not of characteristic 2 . Then, in general, $R D_{2 n}$ is isomorphic to the ring of matrices of this form. This ring of matrices is, in turn, isomorphic to the ring of matrices of the form $\left(\begin{array}{cc}A+B & 0 \\ 0 & -B\end{array}\right)$ with $A, B$ as before. These results are shown in [7].

A matrix of this form is invertible, in an integral domain of characteristic not 2, if and only if $A+B$ and $A-B$ are invertible. Thus, $u \in R D_{2 n}$ is a unit if and only if $A+B$ and $A-B$ are. If $R$ is a field, 
then $u$ is zero-divisor if it is not a unit (Theorem 2.3). Otherwise, it may similarly be determined if $u$ is a zero-divisor.

Any multiplication $x y$ in $R D_{2 n}$ or, equivalently, using the corresponding $R G$-matrices can be done with low-complexity using existing techniques on circulant matrices (such as Fast Fourier Transform methods). $A$ is already circulant, and the operation $x B$ can be done by calculating $x B^{\prime}$ where $B^{\prime}$ is the "flip" of $B$.

6.2.1. Dihedral zero-divisor codes. For a zero-divisor $u \in R D_{2 n}$ where $R$ is a field, finding a basis $S$ for the submodule $W$ can be done by a simple algorithm. Pick, in order, elements from the set $\left\{1, b, b^{2}, \ldots, b^{n-1}\right\}$ until the first $k$ such that $\left\{u, b u, b^{2} u, \ldots, b^{k} u\right\}$ is linearly dependent, or else they are all linearly independent. Then add elements, in order, from $\left\{a, a b, a b^{2}, \ldots, a b^{n-1}\right\}$ until the combined set $\left\{u, b u, \ldots, b^{k-1} u, a u, a b u, \ldots, a b^{l}\right\}$ is linearly dependent. This is shown, by Theorem 7.10, to give maximum rank. An equivalent process can be performed using the $R G$-matrix $U$ directly.

Consider the following code of rate $1 / 2$, constructed from a zero-divisor in $\mathbb{Z}_{2} D_{2 n}$ for $n$ even. Let $u=1+a+a b+\cdots+a b^{n-2}$. Then $v=b+b^{2}+\cdots+b^{n-1}+a b^{n-1}$ satisfies $u v=0$. The $R G$-matrix of $u$ has the form $U=\left(\begin{array}{cc}I_{n} & B \\ B & I_{n}\end{array}\right)$ where $B=\left(\begin{array}{ccccc}1 & 1 & \ldots & 1 & 0 \\ 1 & 1 & \ldots & 0 & 1 \\ \vdots & \vdots & \vdots & \vdots & \vdots \\ 0 & 1 & \cdots & 1 & 1\end{array}\right)$ is a reverse circulant matrix, consisting of all ones in first row except for a zero in the last entry, with subsequent rows determined by the first. It is easy to see that $\operatorname{rank} U=n$.

Now, $V=\left(\begin{array}{ll}E & F\end{array}\right)$. Here, $\operatorname{rank} V=n$. As $\operatorname{rank} U+\operatorname{rank} V=2 n, v$ is a check element for the code when the submodule has dimension $n$, and the code generated is an ideal in $\mathbb{Z}_{2} D_{2 n}$.

This code has length $2 n$ and dimension $n$, and thus rate $1 / 2$. The encoding $\mathbb{Z}_{2}^{n} \rightarrow \mathbb{Z}_{2}^{2 n}$ has a generator and a check matrix $\left(\begin{array}{ll}I_{n} & B\end{array}\right)$ and $E^{\mathrm{T}}$ respectively. A generator matrix of the form $\left(I_{n} B\right)$, where $B$ is a reverse circulant matrix, is known as reverse circulant generator matrix [5]. It is thus clear that codes with these generator matrices always arise from elements in $R D_{2 n}$ of the form $1+a u$, where $u$ can be considered an element from $R C_{n}$.

Now consider the following example of codes over $D_{2 n}$ built up from elements in $C_{n}$. Let $u \in R C_{n}$ be a zero-divisor such that $u v=0$ where $C_{n}$ has generating element $b$. From it we can construct a zero-divisor of the form $u+a x u \in D_{2 n}$ for any $x \in R C_{n}$ with $(u+a x u)\left(v+a y v^{\mathrm{T}}\right)=0$ for any $y \in R C_{n}$. For simplicity, we restrict ourselves to the case $x=y=1$. Additionally, any element $u \in \mathbb{Z}_{2} C_{n}$ (including units) will produce a zero-divisor in $\mathbb{Z}_{2} D_{2 n}$ of the form $u+a u$.

This simple construction can produce surprisingly decent codes. Consider $u=1+b^{2}+b^{5} \in \mathbb{Z}_{2} C_{7}$, which produces the Hamming $(7,4,3)$ code. The zero-divisor $u+a u$ has a corresponding $R G$-matrix with rank 7 and produces a $(14,7,4)$ code which is the best possible for this length and dimension. Similarly $u=1+b^{2}+b^{3}+b^{9}+b^{10}+b^{11}$ produces a $(24,11,8)$ code - also the best distance possible for $(24,11)$ binary code. For $u \in \mathbb{Z}_{2} C_{31}$ given by $1+g+g^{6}+g^{9}+g^{10}+g^{14}+g^{15}+g^{16}+g^{17}+g^{19}+g^{20}+g^{21}+g^{22}+g^{23}+g^{25}+g^{27}$ yields a $(62,30,12)$ code over $\mathbb{Z}_{2} D_{62}$ which has the same distance as the best-known $(62,30)$ code (it could be possible codes with better distance may exist).

6.3. LDPC codes. In general, a code is an LDPC code if its check matrix is sparse, with few non-zero entries. Thus, a code from a group ring encoding will be LDPC if the check element is short, namely contains few terms. LDPC unit-derived codes are obtained by finding a unit element $u \in R G$ so that either $u$ or $u^{-1}$ has only a small number of non-zero coefficients relative to the size of the group. It is then possible to describe whole series of such codes from group rings.

Although most LDPC codes have been produced by randomised techniques, there has been recent activity in the area of algebraic constructions $[13,11,14]$. The group ring encoding framework is a useful tool in this direction, as it seems (as we shall show) that some of these constructions are implicitly working in a group ring. Algebraic constructions have some advantages, including potentially the ability to have immediate generator matrices (it often proves difficult to find one from a randomly produced check matrix) and ascertain performance without extensive testing. One could also envisage a hybrid whereby a random construction is performed within the parameters of an algebraic construction.

6.3.1. Unit-derived Example. LDPC unit-derived codes with no short cycles can be achieved. These are neither cyclic nor an ideal. Consider for example the unit-derived code with check element $v=1+g^{n-1}+$ 
$g^{n-3}+g^{n-8}+g^{n-12}$ in $\mathbb{Z}_{2} C_{1000}$. We omit the generator element $u$ as it has 481 non-zero elements (it is quickly calculated using the Extended Euclidean Algorithm).

Let $W$ be the module generated by $\left\{1, g^{2}, g^{4}, \ldots,\right\}$ in $\mathbb{Z}_{2} C_{1000}$. Then the generator matrix for the code is the matrix obtained by taking its first, third, etc. rows from the $R G$-matrix of $u$ and the check matrix, which will be sparse, is obtained by deleting the first, third etc. columns of the $R G$-matrix of the check element $v$ and then transposing.

6.3.2. Zero-divisor Examples. The regular $(j, k)$ LDPC codes from [14], called quasi-cyclic (QC) LDPC codes, are algebraically constructed. Interestingly, the code can be obtained by shortening a code from an encoding in the group ring $\mathbb{Z}_{2} G$ where $G=C_{m} \times C_{k} \times C_{j}$ i.e. the resultant parity-check matrix for code of block length $m k$ can be obtained from say $v \in R G$. For simplicity, take the case $j=2, k=3$, (a generalisation is straightforward). Let $C_{3}$ and $C_{2}$ have generators $a$ and $b$ respectively. Noting that $\mathbb{Z}_{2} G \cong\left(\mathbb{Z}_{2} C_{m}\right)\left(C_{3} \times C_{2}\right)$ one can write the $R G$-matrix in this group ring:

$$
V=\left(\begin{array}{cccccc}
I_{1} & I_{a} & I_{a^{2}} & I_{b} & I_{a b} & I_{a^{2} b} \\
I_{a^{2}} & I_{1} & I_{a} & I_{a^{2}} & I_{b} & I_{a b} \\
I_{a} & I_{a^{2}} & I_{1} & I_{a b} & I_{a^{2} b} & I_{b} \\
I_{b} & I_{a b} & I_{a^{2} b} & I_{1} & I_{a} & I_{a^{2}} \\
I_{a^{2} b} & I_{b} & I_{a b} & I_{a^{2}} & I_{1} & I_{a} \\
I_{a b} & I_{a^{2} b} & I_{b} & I_{a} & I_{a^{2}} & I_{1}
\end{array}\right)
$$

where each entry is a $m \times m$ circulant permutation matrix determined in the same fashion as [14]. Taking the first 3 elements of every 3rd row gives the parity-check matrix used in [14]. In general, the parity-check matrix will be the same except for row ordering.

As the authors state, the resultant parity-check matrix may not have all linearly independent rows, resulting in a code which may exceed the target rate $1-j / k$ (although only slightly as they discover). Applying knowledge of the group ring may help in achieving the target rate.

The LDPC construction in [11] can also be seen as construction from a group ring, in this case $\mathbb{Z}_{2}\left(C_{N} \times\right.$ $C_{S}$ ), resulting in a parity-check matrix from the 1 st $m N$ rows of the $R G$-matrix, and each block $N \times N$ matrix is a circulant permutation matrix.

Inspired by the above constructions, we propose a regular $(m k, j, k)$ LDPC code with target rate $1-j / k$, from a group ring $R(G \times H)$, where $G=\left\{g_{1}, g_{2}, \ldots, g_{m}\right\}$ and $H=\left\{h_{1}, h_{2}, \ldots, h_{k}\right\}$ are groups of order $m$ and $k$ respectively $(m>k>j)$.

This can be from either a zero-divisor or unit code. The $R G$-matrix from $R(G \times H)$ group ring is a $k \times k$ block matrix where each block is an $m \times m R G$-matrix in $R G$.

Recall that $G \times H=\{(g, h): g \in G, h \in H\}$. Over $Z_{2}$, construction amounts to deciding which group elements to choose from all possible ones. The general idea is to construct an element in the group ring of weight $k$ by picking, for each $h \in H$, exactly one element in $f(h) \in G$, yielding element $v=\sum_{h \in H}(f(h), h)$ $\left(f: H \rightarrow G\right.$ is not necessarily $1-1$ so it is possible that $f\left(h_{i}\right)=f\left(h_{j}\right)$ for $i \neq j$ ). The subsequent $R G$-matrix is,

$$
V=\left(\begin{array}{ccccc}
I_{h_{1}} & I_{h_{2}} & \ldots & \ldots & I_{h_{k}} \\
I_{h_{2}^{-1} h_{1}} & I_{h_{1}} & \ldots \ldots & I_{h_{2}^{-1} h_{k}} \\
\ldots \ldots \ldots \ldots \ldots & \ldots \ldots \ldots & \ldots \\
I_{h_{k}^{-1}} & I_{h_{k}^{-1} h_{2}} & \ldots \ldots \ldots & I_{h_{1}}
\end{array}\right)
$$

Each block matrix $I_{h_{l}}$ has entries $\alpha_{s t}=1$ if $g_{s}^{-1} g_{t}=f\left(h_{l}\right)$ and zero otherwise. $V$ thus has exactly $k$ ones in each row and column.

The submodule $W$ for this code is chosen to have dimension $m j ; j$ rows of block matrices are picked from $V$, resulting in a parity check matrix $C$. Since each $I_{h_{l}}$ has exactly one 1 in each column, $C$ will have $k$ ones in each column and $j$ ones in each row, and is thus a check for a regular $(j, k)$ LDPC code.

For a given $k$ and $m$ there are many possible $j$. An exact rate $1-j / k$ can be achieved when $v$ is a unit. When $v$ is a zero-divisor, then it can be attained whenever a judicious choice of basis $S \subset G \times H$ is possible such that $S v$ is linearly independent (see Section 4.1). 
Initial results are promising, including, over $\mathbb{Z}_{2}\left(C_{k} \times C_{m}\right)$ for $j=3$ and $k=4,(31,26,3)$ and $(21,5,10)$ binary codes which have the best possible distance possible in their class.

\section{Some Relationships with known codes.}

7.1. Cyclic codes. We show here that cyclic codes are exactly zero-divisor codes in group rings on cyclic groups for special cases of the module $W$. Also noteworthy is that Reed-Muller codes are extended cyclic codes and have been shown to be associated with the group ring of the elementary abelian 2-group [4].

For a given polynomial $h(g)$ over a ring $R$, let $r(g)$, be the polynomial of minimal degree such that $h(g) r(g) \equiv 0 \bmod \left(g^{n}-1\right)$ (whenever it exists). Then, a cyclic code over $R$ is generated by $h(g)$ with corresponding check polynomial $r(g)$.

The group ring $R C_{n}$ of the cyclic group $C_{n}$ over a ring $R$ is isomorphic to $R[g] /\left\langle g^{n}-1\right\rangle$. This is a well-known result (e.g. [2]). Cyclic codes of degree $n$ are given by zero-divisors in $R C_{n}$ and the check matrices the counterpart of the zero-divisor.

Theorem 7.1. Let $h(g)=\alpha_{0} 1+\alpha_{1} g+\ldots+\alpha_{r} g^{r}$.

$h(g)$ is the generator polynomial of a cyclic code of length $n$ if and only if the RG-matrix $M\left(R C_{n}, h(g)\right)=\sigma(h(g))$ is a zero divisor in $R_{n \times n}$.

The check matrix of the code is given by the polynomial or group ring element $r(g)$ such that $h(g) r(g) \equiv 0$ $\bmod \left(g^{n}-1\right)$.

Proof. If $h(g)$ is a zero-divisor in $R(G)$ then $h(g) r(g)=l(g)\left(g^{n}-1\right)$ as polynomials.

The code generated by $h(g)$ is the same as the one generated by $d(g)=\operatorname{gcd}\left(h(g), g^{n}-1\right)$ and then $d(g) p(g)=g^{n}-1$.

$M(R G, d(g))$ gives the generator matrix of the code and $M(R G, p(g))$ the check matrix.

Cyclic codes in standard notation are presented using non-square matrices. However, the matrices can be made square by considering them as group ring elements.

In cyclic codes, the matrix representation of $u$ is used and the mapping is given as $x \mapsto x u$ where $x \in R^{r}$ and $u \in R_{r \times n}$. Consider $x$ as an element in $R^{n}$ (by adding 0's) and the element $u$ as an element in $R_{n \times n}$ which is the group ring completion of the matrix $u$. Then the mapping is $x \mapsto x u$ in matrix form and is equivalent to the previous mapping.

See also Section 7.3 on ideals in the cyclic group ring.

7.2. Complexity relationship to standard mappings. Codes from group ring encodings are not complex to implement. Since the first row of an $R G$-matrix specifies the entire matrix, any operations on them can be quickly performed.

Normally, codes are considered maps $\beta: R^{r} \rightarrow R^{n}$ with $r<n$. The codes from group ring encodings are $x \mapsto x u$ and these have equivalent encodings in matrix form $R_{n \times n} \rightarrow R_{n \times n}$ given by $\alpha: X \mapsto X U$. Now $X$ is an $R G$-matrix with 0 in $n-r$ entries of its first row and $X$ and $X U$ are determined by their first rows. Thus the mappings $\beta$ and $\alpha$ require the same number of calculations.

7.3. Ideals. Here we discuss conditions under which codes as defined in Section 3 are ideals. It transpires that unit-derived codes as defined in Section 5 are never ideals and that zero-divisor codes as defined in Section 4 are ideals only in very special cases.

DEFINITION 7.2. I is said to be an ideal in the ring $H$ if $I$ is a subring such that (i) hi $\in I, \forall h \in H, \forall i \in I$ and (ii) $i h \in I, \forall h \in H, i \in I$. Such an ideal is often referred to as a two-sided ideal. I is said to be a left ideal in $H$ if $I$ is a subring such that $h i \in I, \forall i \in I, \forall h \in H$. I is said to be a right ideal in $H$ if $I$ is a subring of $H$ such that $i h \in I, \forall i \in I, \forall h \in H$.

In the case of a commutative ring there is no distinction between left, right and two-sided ideals.

The cyclic codes are ideals in the group ring on the cyclic group but for example the quasi-cyclic and shortened cyclic codes are not ideals. Our intention here is to clarify the situation with respect to codes in group rings as defined in Section 3.

Recall that such a code is either $W u$ or $u W$ where $W$ is a submodule of $R G$ and $u \in R G$. We consider the right encoding $W u$; the other one is similar. Assume that $G=\left\{g_{1}, g_{2}, \ldots, g_{n}\right\}$ and here consider cases where the ring $R$ is a field. 
Assume also that $W$ is generated by $S=\left\{g_{i_{1}}, g_{i_{2}}, \ldots, g_{i_{r}}\right\}$. The code is then $\mathcal{C}=\{x u: x \in W\}$ and is generated by $S u=\left\{g_{i_{1}} u, g_{i_{2}} u, \ldots, g_{i_{r}} u\right\}$. We may assume as explained in Section 4 that $S u=$ $\left\{g_{i_{1}} u, g_{i_{2}} u, \ldots, g_{i_{r}} u\right\}$ is linearly independent.

The rows of an $n \times n$ matrix $U$ are designated in order by $\underline{u}_{1}, \underline{u}_{2}, \ldots, \underline{u}_{n}$.

Define $G_{j}$ to be the $R G$-matrix corresponding to the group element $g_{j} \in G$. Thus $G_{j}$ is the matrix whose first row has a 1 in the $j^{t h}$ position and zeros elsewhere and this first row determines the $R G$-matrix $G_{j}$. It then follows $G_{j} U$ is the $R G$-matrix with first row $\underline{u}_{j}$.

Lemma 7.3. Suppose $g_{i_{1}} u, g_{i_{2}} u, \ldots, g_{i_{r}} u$ is linearly independent. Then $\operatorname{rank} U \geq r$.

Proof. Since $\left\{g_{i_{1}} u, g_{i_{2}} u, \ldots, g_{i_{r}} u\right\}$ is linearly independent so is $\left\{G_{i_{1}} U, G_{i_{2}} U, \ldots, G_{i_{r}} U\right\}$. Thus $\underline{u}_{i_{1}}, \underline{u}_{i_{2}}, \ldots, \underline{u}_{i_{r}}$ is linearly independent and hence $U$ contains $r$ linearly independent rows. $\square$

TheOREM 7.4. Let $\mathcal{C}$ be the code $W u$ with $W$ generated by $S=\left\{g_{i_{1}}, g_{i_{2}}, \ldots, g_{i_{r}}\right\}$ such that $S u=$ $\left\{g_{i_{1}} u, g_{i_{2}} u, \ldots, g_{i_{r}} u\right\}$ is linearly independent. Then $\mathcal{C}$ is a left ideal if and only if $\operatorname{rank} U=r$.

Proof. Suppose $\operatorname{rank} U=r$. We wish to show that $W u$ is an ideal in $R G$. It will be sufficient to show that $g u$ is in $\mathcal{C}$ for any $g \in G$.

Since $g_{i_{1}} u, g_{i_{2}} u, \ldots, g_{i_{r}} u$ is linearly independent then by Lemma 4.6 of Section $4 G_{i_{1}} U, G_{i_{2}} U, \ldots, G_{i_{r}} U$ is linearly independent and so the rows $\underline{u}_{i_{1}}, \underline{u}_{i_{2}}, \ldots, \underline{u}_{i_{r}}$ of $U$ are linearly independent. Since $U$ has rank $r$ these rows thus form a basis for the row space of $U$.

Suppose then $g \in G$. If $g \in S$ then clearly $g u \in \mathcal{C}$.

Suppose $g \notin S$. Then $g=g_{j}$ where $j$ is not any of $i_{1}, i_{2}, \ldots, i_{r}$. Now $G_{j} U$ is the $R G$-matrix whose first row is $\underline{u}_{j}$, the $j^{\text {th }}$ row of $U$.

Then $\underline{u}_{j}=\sum_{k=1}^{r} \alpha_{k} u_{i_{k}}$ for some $\alpha_{k} \in R$ since the rows in the sum are a basis for the row space of $U$. Then by Lemma 4.6 of Section 4 it follows that $G_{j} U=\sum_{k=1}^{r} \alpha_{k} G_{i_{k}} U$. Hence $g_{j} u=\sum_{k=1}^{r} \alpha_{k} g_{i_{k}} u$ and thus $g_{j} u \in \mathcal{C}$ as required.

Suppose on the other hand $\mathcal{C}$ is a left ideal. Then $g_{i} u \in \mathcal{C}$ for the $i^{t h}$ element $g_{i} \in G$. Then as before $\underline{u}_{j}$ is a linear combination of $\underline{u}_{i_{1}}, \underline{u}_{i_{2}}, \ldots, \underline{u}_{i_{r}}$. Hence $\operatorname{rank} U \leq r$. Since $\underline{u}_{i_{1}}, \underline{u}_{i_{2}}, \ldots, \underline{u}_{i_{r}}$ is linearly independent it follows that $\operatorname{rank} U=r$. $\square$

Note that the proof also shows that when $\mathcal{C}$ is a left ideal, then $\mathcal{C}=W u=R G u$.

If $u$ is a unit then $\operatorname{rank} U=n$. Hence $\mathcal{C}$ is not an ideal in a unit-derived code - the module $W$ is never equal to all of $R G$ in our definition of unit-derived code.

Let $I$ be a left ideal in a ring $R$. Similar remarks apply to right and two-sided ideals. If $I$ contains a unit then clearly $I$ contains the identity and hence $I=R$. Now $I$ is said to be a proper ideal if $I \neq 0$ and $I \neq R$. Here again we can see that a unit-derived code is never an ideal as this has the form $W u$ where $u$ is a unit and thus $W u$ has a unit.

Suppose $I$ is a proper left ideal of a group ring $R G$ where $R$ is a field. Then $I$ is a subspace of $R G$ and so is generated as an $R$-module by $u_{1}, u_{2}, \ldots, u_{s}$ with $u_{i} \in R G$. Since $I$ is a proper ideal none of the $u_{i}$ can be units and so by Theorem 2.3 each $u_{i}$ is a zero-divisor. Also no linear combination of the $u_{i}$ can be a unit as $I$ does not contain any unit. Thus:

THEOREM 7.5. I is a proper ideal in a group ring $R G$, with $R$ a field, if and only if $I$ is generated as a module by $u_{1}, u_{2}, \ldots, u_{s}$ where each $u_{i}$ is a zero-divisor and no linear combination of the $u_{i}$ is a unit.

A left ideal $I$ is principal in $R G$ if and only if it has the form $R G u$ for an element $u \in R G$. Thus proper principal ideals in the group ring over a field are of the form $R G u$ where $u$ is a zero-divisor. These are the particular zero-divisor codes where the rank of $U$ equals the number of elements in the generating set $S$ of $W$ where $S u$ is linearly independent.

7.3.1. Ideals in cyclic group rings. Because of its particular nature as a polynomial type ring, it is easy to show that all ideals in the cyclic group ring are principal.

LEMMA 7.6. Every ideal in the group ring of the cyclic group is principal.

Proof. Let $I$ be an ideal in the group ring of the cyclic group $R G$ where $G$ is generated by $g$. Choose $f(g)$ in $I$ of minimal degree in $g$. Let $x(g) \in I$. Then by Division Algorithm to polynomials, $x(g)=q(g) f(g)+r(g)$ 
where $r(g)=0$ or $r(g)$ has degree less than $f(g)$. Now $r(g) \in I$ since $x(g) \in I$ and $f(g) \in I$. Since $f(g)$ is of minimal degree in $I$ this implies $r(g)=0$ and $x(g)=q(g) f(g)$. Hence $I$ is a principal ideal generated by $f(g)$.

Suppose now we have a zero-divisor $u$ in the cyclic group $\operatorname{ring} R G$ and that $\operatorname{rank} U=r$. In order for $u$ to be a principal zero-divisor we require an element $v \in R G$ such that $u v=0$ and $\operatorname{rank} V=n-r$. Such a $v$ always exists in the cyclic group ring. Choose $v$ to be an element of least degree such that $u v=0$. Here in fact what we are doing is choosing a generator for the annihilator of $u$ which is a principal ideal.

THEOREM 7.7. Suppose $u$ is a zero-divisor in the cyclic group ring $R G$ with $G$ of order $n$. Let $v$ be an element of least degree such that $u v=0$. If $\operatorname{rank} U=r$ then $\operatorname{rank} V=n-r$.

Proof. If $u v_{1}=0$ then by Division Algorithm, $v_{1}=q v+r$ where $r=0$ or $\operatorname{deg}(r)<\operatorname{deg}(v)$. Then multiplying through by $u$, and noting that elements commute, we see that $u r=0$. Since $v$ is of least degree such that $u v=0$ this implies that $r=0$ and hence $v_{1}=q v$.

We now show that the null-space of $U$ is generated by the rows of $V$. The null-space of $U$ is the set of all vectors $\underline{x}$ such that $U \underline{x}=0$.

Suppose now $\underline{x}$ is a vector in the null-space of $U$, so that $U \underline{x}=0$. Let $T$ be the $R G$-matrix with first row $\underline{x}$. Now $\underline{x}$ determines $T$. Then $U T$ is an $R G$-matrix with first row 0 and hence $U T=0$. Therefore $u t=0$ in the group ring. Hence $t=q v$ for some $q \in R G$. Therefore $T=Q V$. Hence the rows of $T$ are linear combinations of the rows of $V$. In particular $\underline{x}$ is a linear combination of the rows of $V$. Thus the rows of $V$ generate the null-space of $U$. Since by linear algebra the null-space of $U$ has dimension $n-r$ it follows that $V$ has rank $n-r$.

Suppose $u$ is a zero-divisor in the group ring $R G$, with $G$ generated by $g$, and that as polynomials $u v=g^{n}-1,{ }^{5}$ then $v$ as an element of $R G$ has the property that it is of minimal degree such that $u v=0$. It follows that $\operatorname{rank} U+\operatorname{rank} V=n$.

Corollary 7.8. If $u v=g^{n}-1$ then $\operatorname{rank} U+\operatorname{rank} V=n$.

7.4. Rank from cyclic and dihedral zero-divisors. Suppose $u$ is a zero-divisor in the group ring $R G$. In the cyclic group ring case when $\operatorname{rank} u=r$ and $U$ is obtained from the natural listing of $G$, the first $r$ elements $u, g u, \ldots, g^{r-1} u$ in the group ring and the the first $r$ rows of $U$ give the (full rank) zero-divisor codes. This is expressed in the following theorem.

TheOREm 7.9. Let $R G$ be a group ring where $R$ is a field and $G$ the cyclic group $\left\{1, g, g^{2}, \ldots, g^{n}\right\}$. Suppose $u \in R G$ is a zero divisor. Let $r$ be the first value such that $\left\{u, g u, g^{2} u, \ldots, g^{r} u\right\}$ is linearly dependent. Then $\operatorname{rank}(u)=r$.

Proof. Linear dependence means that, for some $\alpha_{i} \in R, i=1, \ldots, r-1$ that,

$$
g^{r} u=\alpha_{0} u+\alpha_{1} g u+\alpha_{2} g^{2} u+\cdots+\alpha_{r-1} g^{r-1} u .
$$

By multiplication by $g^{l}$,

$$
g^{r+l} u=\alpha_{0} g^{l} u+\alpha_{1} g^{1+l} u+\alpha_{2} g^{2+l} u+\cdots+\alpha_{r-1} g^{r-1+l} u .
$$

Therefore for every $x=\sum_{i=0}^{n-1} \beta_{i} g^{i} \in R G, x u$ can be written $\sum_{i=0}^{r-1} \beta_{i} g^{i} u$, i.e. in terms of $\left\{u, g u, g^{2} u, \ldots, g^{r-1} u\right\}$.

A similar result holds for the dihedral group.

THEOREM 7.10. Consider the group ring $R D_{2 n}$ where $R$ is a field and $D_{2 n}$ the dihedral group. Let $u \in R G$ and $S^{\prime}$ be the first elements $\left\{1, b, b^{2}, \ldots, b^{k-1}\right\} \subseteq\left\{1, b, \ldots, b^{n-1}\right\}$ such that $\left(S^{\prime} \cup b_{k}\right) u$ is linearly dependent. Subsequently, let $S=S^{\prime} \cup\left\{a, a b, \ldots, a b^{l}\right\}$ be the first set such that $S u$ is linearly dependent. Then $\operatorname{rank}(u)=|S|$.

Proof. By the linear dependence,

$$
b^{k} u=\left(\alpha_{0}+\alpha_{1} b+\cdots+\alpha_{k-1} b^{k-1}\right) u .
$$

\footnotetext{
${ }^{5}$ Cyclic codes are defined using such elements.
} 
By multiplication on the left on both sides, it can be seen that for every $k \leq m<n, b^{m} u$ can be written in terms of the previous $k$ powers of $b$ (times $u$ ) and thus, ultimately, in terms of $S^{\prime}$. Also,

$$
a b^{l} u=\left(\alpha_{0}+\alpha_{1} b+\cdots+\alpha_{k-1} b^{k-1}+\beta_{0} a+\beta_{1} a b \cdots+\beta_{l-1} a b^{l-1}\right) u .
$$

Multiplication on the left by $b^{l-m}$ yields that

$$
\begin{aligned}
a b^{m} u=\left(\alpha_{0} b^{l-m}\right. & +\alpha_{1} b^{l-m+1}+\cdots+\alpha_{k-1} b^{l-m+k-1} \\
& \left.+\beta_{0} a b^{m-l}+\beta_{1} a b^{m-l+1}+\cdots+\beta_{l-1} a b^{m-1}\right) u .
\end{aligned}
$$

Thus, for each $l \leq m<n, a b^{m} u$ can be written in terms of the form $b^{i} u$ (which we showed above can be written in terms of the first $k$ powers of $b$ ), and in terms of the previous $l$ elements of the form $a b^{i}$.

8. Conclusions. We have described a method for producing codes from units and zero-divisors in group rings, including, vitally, a method to obtain generator and check matrices for them.

It is our belief that this is an intuitive method which has already shown promise even in the example codes presented. The framework provides a basis to anyone wishing to further develop or investigate these codes. The rich synergy of matrix algebra and group ring structure shows itself to be a powerful new method.

We note that it is possible to obtain convolutional codes from unit-derived and zero-divisor codes from group rings. When a ring $R$ has zero-divisors then it is possible to obtain units in a group ring $R G$ when the group $G$ is infinite, such as $G=C_{\infty}$ (the infinite cyclic group), and from these units to derive convolutionaltype codes. Results of this will appear as future work.

The underlying algebraic structure of group rings often allows the calculation of distance directly. The description as an encoding enables one at times to work within the group ring to, for example, calculate the minimum distance of a code or to make a code with a certain minimum distance.

Also of note is that codes over the integers can be constructed using known units in $\mathbb{Z} G$. These include alternating units, Bass units, Hoeschmann Units, bicyclic units and others, the details of which may be found in [12]. Other future work we envisage includes the generalisation to consider combinations of group ring encodings acting in unison to produce a code.

\section{REFERENCES}

[1] Louay M.J. Bazzi And Sanjoy K. Mitter, Some constructions of codes from group actions, Preprint. Under Submission, (2003).

[2] R. E. Blahut, Algebraic codes for data transmission, Cambridge University Press, 2003.

[3] P. J. Davis, Circulant Matrices, John Wiley and Sons, Inc., New York, 1979.

[4] JR E. F. Assmus, On Berman's characterization of the Reed-Muller codes, Journal of Statistical Planning and Inference, 56 (1996), pp. 17-21.

[5] W. C. Huffman and V. Pless, Fundamentals of error-correcting codes, Cambridge Univ. Press, 2003.

[6] G. Hughes, Constacyclic codes, cocycles and $a u+v-u-v$ construction, IEEE Trans. Inform. Theory, 46 (2000), pp. 674680.

[7] Ted Hurley, Group rings and rings of matrices, Inter. J. Pure \& Appl. Math., (2006), pp. 319-335.

[8] F. J. MacWilliams, Codes and ideals in group algebras, Combinatorial Mathematics and its Applications, (1969), pp. 312328.

[9] - Orthogonal circulant matrices over finite fields, and how to find them, J. Comb. Theory, 10 (1971), pp. 1-17.

[10] F. J. MacWilliams and N.J.A. Sloane, The Theory of Error-Correcting codes, North-Holland Amsterdam/London/New York/Tokyo, 1998.

[11] O. Milenkovic, I. DJordjevic, And B. VAsic, Block-circulant low-density parity-check codes for optical communication systems, IEEE Journal of Selected Topics in Quantum Electronics, 10 (2004), pp. 294-299.

[12] César P. Milies and Sudarshan K Sehgal, An Introduction to Group Rings, Klumer, Dordrecht/Boston/London, 2002.

[13] H. Tan, Jun Xu, Yu Kou, Shu Lin, and Khaled A. S. Abdel-Ghaffar, On algebraic construction of gallager and circulant low-density parity-check codes, IEEE Trans. Inform. Theory, (2004), pp. 1269-1279.

[14] R.M. Tanner, D. Sridhara, A. Sridharan, T.E. Fuja, and Jr. Daniel Costello, Ldpc block and convolutional codes based on circulant matrices, IEEE Trans. Inform. Theory, (2004), pp. 2966-2984.

[15] Harold N. Ward, Quadratic residue codes and divisibility, Handbook of Coding Theory, Elsevier, Amsterdam, 1998, ch. 9 , pp. $827-870$. 